\title{
Introduction
}

Romi Kher' / Thomas S. Lyons ${ }^{2}$

\section{University-based Venture Development Organizations}

\author{
${ }^{1}$ Baruch College Zicklin School of Business, New York, NY 10010, USA, E-mail: Romi.Kher@baruch.cuny.edu \\ ${ }^{2}$ University of Tennessee at Chattanooga, 545 Oak Street, Chattanooga, TN 37403-2504, USA, E-mail: Thomas-Lyons@utc.edu
}

DOI: 10.1515/erj-2020-0057

In this special issue, we have curated two editorials and four articles that address the topic of university-based efforts to support the commercialization of intellectual property through entrepreneurship. While the focus is on the institutional players in these efforts - Venture Development Organizations (VDOs) - the insights shared range from observations regarding the academic entrepreneurs served by VDOs, to the VDOs themselves, to the larger ecosystems of which both are a part and their elements. The perspective presented is international in scope, reflecting experiences from Egypt, Spain, South Africa, Taiwan and the United States.

In the opening editorial, Swartz, Marks and Scheepers discuss the dynamism of the South African entrepreneurial ecosystem, with its growing number and types of participants, including multiple universities. These authors observe that this type of activity is fertile ground for research, which is much needed and currently underdeveloped. In the second editorial, Halbinger discusses the potential value of makerspaces to the university-based innovation ecosystem and calls for deeper research into this element of such an ecosystem.

Blair and Shaver choose to examine the university-based VDO phenomenon from the perspective of the faculty who elect to become entrepreneurs. The authors' US-based research sheds light on what motivates these academics to make this choice, offering important insights to institutions seeking to influence their behavior.

In his article, which studies a university-based accelerator program in Egypt, Ismail offer a framework for the design of an effective accelerator. They note that such a framework cannot be a one-size-fits-all template. It must be tailored to the specifics of its context and to the needs of the entrepreneurs it serves. This makes for an appropriate segue to the two other articles in this special issue, which expand the discussion of context to the full entrepreneurial ecosystem of which the university-based VDO is a part. Hsieh and Kelley, using Taiwan as their geographical base, examine the relationship between the VDO and its ecosystem. They discuss six elements that are crucial to promoting academic entrepreneurship: culture, finance, human capital, market, policy and support. They find that helping these entrepreneurs to understand and reach their market is the most important of these elements to their success, which requires engaging with the community. Finally, Franco-Leal et al. argue that a complete ecosystem is essential to the success of academic spinoff ventures. This is because their research in Spain shows that these ventures need different kinds of support depending on their stage in the business lifecycle. The university is of more value to them during the pre-venture and startup stages, but once they move to the growth stage, networking with others outside the university becomes more valuable to their success.

These valuable insights provide a well-rounded view of the subject of university-based venture development organizations. They advance our knowledge in this arena but also suggest that opportunities for further research abound.

Romi Kher is the corresponding author.

(c) 2020 Walter de Gruyter $\mathrm{GmbH}$, Berlin/Boston.

This content is free. 